\title{
La inteligencia emocional en adolescentes del 5to blanco de secundaria de la Unidad Educativa Tomás Frías de la ciudad de Potosí
}
Inteligência emocional em adolescentes da $5^{a}$ série branca do ensino médio da Unidade Educacional Tomás Frías da cidade de Potosí

\author{
Karin Elizabeth Guerra Delgado \\ karineguerra@outlook.es \\ https://orcid.org/0000-0001-6852-3228 \\ Universidad Privada Domingo Savio, Potosí, Bolivia
}

Recibido: febrero 2021 Revisado: marzo 2021 Aceptado: abril 2021 Publicado: mayo 2021

\section{RESUMEN}

La investigación tuvo como objetivo proponer un programa educativo para desarrollar la inteligencia emocional en adolescentes de 5 to blanco de secundaria de la Unidad Educativa Tomás Frías, de la ciudad de Potosí. Se estudiaron los fundamentos teóricos de la inteligencia emocional teniendo como base a las inteligencias múltiples(Gardner, Goleman, Salovey, Jacson, etc.) Metodológicamente se fundamentó y utilizó el instrumento de nivel empírico la entrevista semiestructurada. Los resultados indicaron que existen dificultades en las dimensiones inter e intrapersonal, siendo que los adolescentes muestran falencias en saber reconocer emociones, comunicar sus sentimientos, reconocer sus logros, admitir sus propios errores y en tomar decisiones sin dejarse influenciar. Por lo tanto, se propone planificar la acción educativa en función de desarrollar la inteligencia emocional, a través de talleres y videos didácticos que orienten la solución posible del problema.

Palabras clave: Inteligencia emocional; adolescentes; escolares; programa
The objective of the research was to propose an educational program to develop emotional intelligence in adolescents from the 5 th grade of secondary school of the Tomás Frías Educational Unit, in the city of Potosí. The theoretical foundations of emotional intelligence were studied based on multiple intelligences (Gardner, Goleman, Salovey, Jacson, etc.) Methodologically, the semistructured interview was based on and used the empirical level instrument. The results indicated that there are difficulties in the inter and intrapersonal dimensions, being that adolescents show deficiencies in knowing how to recognize emotions, communicate their feelings, acknowledge their achievements, admit their own mistakes and make decisions without being influenced. Therefore, it is proposed to plan educational action in order to develop emotional intelligence, through workshops and didactic videos that guide the possible solution of the problem.

Key words:

Intrauterine stimulation; fetal development; prenatal stimulation; program
RESUMO

O objetivo da pesquisa foi propor um programa educacional para o desenvolvimento da inteligência emocional em adolescentes do $5^{\circ}$ ano do ensino médio da Unidade Educacional Tomás Frías, na cidade de Potosí. Os fundamentos teóricos da inteligência emocional foram estudados com base nas inteligências múltiplas (Gardner, Goleman, Salovey, Jacson, etc.). Metodologicamente, a entrevista semiestruturada baseouse e utilizou o instrumento de nível empírico. Os resultados indicaram que existem dificuldades nas dimensões inter e intrapessoal, sendo que os adolescentes apresentam deficiências em saber reconhecer emoções, comunicar seus sentimentos, reconhecer suas realizações, admitir seus próprios erros e tomar decisões sem serem influenciados. Para tanto, propõe-se o planejamento de ações educativas para o desenvolvimento da inteligência emocional, por meio de oficinas e vídeos didáticos que orientem a possível solução do problema.

Palavras-chave: Inteligência emocional; adolescentes; escolares; Programa 


\section{INTRODUCCIÓN}

Está comprobado en algunas investigaciones que la inteligencia emocional aporta cerca del $90 \%$ para el éxito en la vida, frente a un $10 \%$ que aporta la inteligencia racional. Esto no quiere decir que una es más importante que la otra, al contrario, logrando tener ambas inteligencias se podrían tener seres humanos más íntegros, exitosos y asertivos en las comunicaciones.

El filósofo griego Aristóteles muestra claramente la complejidad del manejo de la inteligencia emocional: "Cualquiera puede ponerse furioso... eso es fácil. Pero estar furioso con la persona correcta, en la intensidad correcta, en el momento correcto, por el motivo correcto, y de la forma correcta... no es fácil" (Goleman, 2000, p.13).

Las primeras aproximaciones que hablan sobre la inteligencia emocional hacen referencia al año 1920 cuando Thorndike, citado por Salovey, hace mención a lo que es la inteligencia social, esto incluía "La habilidad de supervisar y entender las emociones propias y las de los demás, discriminar entre ellas y usar la información para guiar el pensamiento y las acciones de uno" (2007, p. 433).

Sin embargo, más adelante el conductismo frenó el estudio de la inteligencia y de las emociones al no considerar a estos como objeto de su estudio. Posteriormente en la década del ochenta la aparición de la teoría de Piaget, si bien no mostró mucho interés por la relación entre el desarrollo de la inteligencia y de la emoción, algunos de sus seguidores sugirieron la necesidad de observar cómo las emociones pueden influir positivamente en el desarrollo de la inteligencia durante la infancia.

Posteriormente Gardner al hablar sobre las inteligencias múltiples, introduce el tema de la inteligencia emocional. Desde el enfoque moderno y más abierto se sugiere que la inteligencia debe describir su relación con elementos como la motivación, la emoción o la personalidad, en vez de limitarla exclusivamente a las aptitudes de la unión de dos palabras tradicionalmente usadas por separado (la inteligencia y la emoción) En su libro Estructuras de la Mente. La Teoría de las Inteligencias Múltiples (1993) define a la inteligencia como: "La capacidad para resolver problemas, o de crear productos, que sean valiosos en uno o más ambientes culturales" (p.5).

Dice él que la Inteligencia Múltiple: “Es un conjunto pequeño de potencialidades intelectuales humanas, que todos los individuos pueden tener en virtud de que pertenecen a esta especie" ( $p$. 216). Hace énfasis en que las inteligencias que posee el ser humano no se limitan a una sola área, que posee ocho inteligencias de las cuales unas predominan más que otras: Lógico - Matemática, Lingüística, Espacial, Musical, Corporal-kinestésica, Intrapersonal, Interpersonal, Naturalista.

La inteligencia interpersonal e intrapersonal juegan un papel importante en la construcción de la inteligencia emocional, comprendida como la capacidad de reconocer nuestros propios sentimientos y los ajenos, para saberlos manejar y generar mejores resultados en las competencias sociales.

Para debatir en el marco de una problemática vigente del campo de la psicología escolar. El presente trabajo se elaboró con base a una observación realizada durante la ejecución de la práctica profesional en la Unidad Educativa Tomás Frías, específicamente en el curso 5to Blanco de secundaria. La investigadora evidenció durante un taller realizado el escaso compañerismo en los estudiantes y la falta de empatía por el otro, presenciando, incluso, la falta de respeto en un ambiente hostil con burlas y poca comunicación verbal. A futuro serán ellos quienes no podrán alcanzar un equilibrio emocional, tendrán baja 
autoestima, desencadenarán relacionesconflictivas, serán inseguros a la hora de tomar decisiones; lo cual traerá como consecuencia una posible depresión. Por otra parte serán problemáticos y vulnerables ante cualquier situación, rechazados socialmente por sus conductas negativas, tendrán un bajo aprovechamiento escolar el cual podría llevarlos muchas veces al abandono del aula. Es así que el problema principal detectado, fue el inadecuado manejo de la inteligencia emocional, como una de las causas de la conducta que muestran los adolescentes.

A partir de ello la investigación se plantea la siguiente interrogante: ¿Será posible crear un programa educativo para desarrollar la inteligencia emocional, en los adolescentes de 5to blanco de secundaria de la Unidad Educativa Tomás Frías de la ciudad de Potosí, con el fin de mejorar las relaciones con sus familiares y compañeros de curso, para crear un ambiente mejor tanto en aula como fuera de ella?

Se justifica esta investigación pues toda persona debe tener desarrollada la inteligencia emocional ya que es ésta la que la impulsa a estar siempre motivada, con mayor autoestima y predispuesto siempre al diálogo, la que le permitirá tomar decisiones adecuadas a la hora de afrontar cualquier situación que se le presente. La inteligencia emocional está íntimamente relacionada con las emociones, su control y manejo para sacarle el mayor provecho; hacerlo o no facilita a futuro el éxito o el fracaso del adolescente.

Goleman expresa que:

La inteligencia emocional incluye la habilidad para percibir con precisión, valorar y expresar emoción; la habilidad de acceder y/o generar sentimientos cuando facilitan pensamientos; la habilidad de comprender la emoción y el conocimiento emocional; y la habilidad para regular las emociones para promover crecimiento emocional e intelectual. (1998, p.125).

Goleman (1998), autor del libro La práctica de la inteligencia emocional, define, define la inteligencia emocional como: "La Capacidad de reconocer nuestros propios sentimientos, los sentimientos de los demás, motivarnos y manejar adecuadamente las relaciones que sostenemos con los demás y con nosotros mismos" (1998 p.196).

Un buen desarrollo de la inteligencia emocional permite a las personas entender mejor el mundo en el que viven y a los otros seres humanos que habitan en él. Pero sobre todo consigue que la persona se conozca mejor a sí mismo, comprenda y sea capaz de dirigir su vida y ayudar al otro a llevar y afrontar sus problemas de la mejor manera, para así ser capaz se sentirse bien consigo y con el otro logrando una vida satisfactoria y plena.

\section{Perspectiva teórica}

Refiere Salovey que la inteligencia emocional es:

La habilidad para percibir con precisión, valorar y expresar emociones, relaciona también la habilidad para acceder y/o generar sentimientos cuando facilitan el pensamiento, también la habilidad para entender emoción y conocimiento emocional y la habilidad para regular emociones que promuevan el crecimiento emocional e intelectual (2007, p.443).

En la cita se menciona que las investigaciones apuntan a que la inteligencia emocional va acompañada de lo intelectual, pero mucho más allá de una inteligencia estos autores hacen referencia a la habilidad para percibir el hecho de entender y manejar la parte emotiva del ser humano. 
Para Goleman la inteligencia emocional es: "La capacidad de reconocer nuestros propios sentimientos y los ajenos, de motivarnos y de gestionar las emociones, en nosotros mismos y en nuestras relaciones" (2000, p. 41).

Esta definición dada por Goleman hace referencia al autoconocimiento y en base a esto se buscar ser mejor, encontrando las debilidades y fortalezas primero en uno mismo y de esta manera mejorar las relaciones interpersonales. Por otro lado indica que la inteligencia emocional está interrelacionada con las competencias sociales y las emociones. Es la habilidad y los factores que determinan la eficacia con la que uno se entiende a sí mismo, se expresa, entiende a los demás y cómo lidia con las demandas diarias.

Siguiendo la concepción de la inteligencia emocional, Jacson al respecto expresa:

Es la capacidad para procesar la información emocional con exactitud y eficacia, lo que incluiría habilidades para percibir, asimilar, comprender y regular, tanto las emociones como los estados de ánimo de mayor duración. Este control o regulación de las propias emociones y estados de ánimo ha sido considerado por un tercer componente de la empatía, que completaría los dos aspectos, afectivo y cognitivo (2006, pp.45-46).

Enmarcadas dentro de las inteligencias múltiples se encuentran las inteligencias intra e inter personales. Gardner define la inteligencia intrapersonal como:

La capacidad medular que opera aquí es el acceso a la propia vida sentimental, la gama propia de efectos o emociones: la capacidad para para efectuar al instante discriminaciones entre estos sentimientos $y$, con el tiempo, darles un nombre, desenredarlos en códigos simbólicos, de utilizarlos como un modo de comprender y guiar la conducta propia (2001, p.189).

Por lo tanto, la inteligencia intrapersonal es la inteligencia para que uno mismo constituya el conjunto de capacidades que permiten formar un modelo fiel y positivo de sí mismo.

Por otra parte Gardner define a la inteligencia interpersonal como:

...es la habilidad para notar y establecer distinciones entre otros individuos y, en particular, entre sus estados de ánimo, temperamentos, motivacionales e intencionales...comprende la capacidad del infante para discriminar entre los individuos a su alrededor y para descubrir sus estados de ánimo (2001, p.189).

Esta inteligencia es la que permite comprender a los demás, teniendo en cuenta sus distintos estados de ánimo y puntos de vista de un tema en común. Es la capacidad que se tiene para ayudar a otros sin pedir o esperar nada a cambio.

\section{La adaptabilidad}

Incluye las habilidades del uso de vocabulario apropiado y expresar verbalmente las emociones en la manera en que sean comunes en la propia cultura. Conforme la madurez, se adquieren los signos característicos de la cultura que vinculan las habilidades emocionales con el rol social, y la habilidad para adaptarse y afrontar las emociones aversivas 0 estresantes utilizando estrategias autorreguladoras.

\section{El área del estado de ánimo en general}

Se trata de alcanzar la capacidad emocional de autoeficacia, la persona se percibe a sí misma del modo como realmente desea sentirse. La 
autoeficacia emocional significa que se aceptan las experiencias emocionales únicas y excéntricas o culturalmente convencionales. La aceptación está ligada con las creencias del individuo acerca de lo que constituye un balance emocional deseado y, en esencia, vivir de acuerdo con las propias teorías de la emoción que están integradas al sentido moral.

\section{Inteligencia emocional en adolescentes}

La adolescencia es una de las etapas más complejas de los seres humanos. Es también la etapa en la que el individuo construye su identidad a través de sus experiencias y aprendizajes, determinando así las características que lo definirán en el futuro como persona y lo encauzarán hacia el éxito o el fracaso; sin lugar a dudas, las emociones y el autoconocimiento son esenciales en este proceso.

Fernández sustenta que:

La inteligencia emocional de los adolescentes va de la mano con el desarrollo psicosocial, como lo describe Erikson. Si el desarrollo del adolescente cumple con los criterios esperados, está desarrollando una inteligencia emocional adecuada a su edad, indican que la niñez intermedia, entre los 6 años y la pubertad, es una etapa en la cual se consolida el aprendizaje de las habilidades sociales, el auto concepto y la comprensión de las emociones, ya sea de manera adecuada o inadecuada (2011, p. 89).

En la etapa psicosocial de los niños entre los siete años y la pubertad, se encuentran la laboriosidad frente a la inferioridad, según Erickson mencionado por Papalia (2009) La característica principal de esta etapa es que los niños inician, desarrollan y terminan sus proyectos. Si esta actividad se desarrolla con éxito, los niños comienzan a sentirse orgullosos de sus logros y sienten confianza en sus capacidades. Si esta etapa fracasa, el niño comienza a sentirse inferior, duda de sus capacidades y se frustra.

Entre la pubertad y la adultez temprana, los adolescentes se encuentran en la etapa de identidad frente a confusión de identidad. Esta etapa es importante ya que en ella es cuando se consolida la personalidad y el auto concepto e independencia. Durante este periodo los adolescentes conforman su identidad a través de la elección de la ocupación, adopción de valores y el desarrollo de una identidad sexual satisfactoria.

Diserta Bisquerra:
Para poder resolver estos problemas de manera adecuada, los adolescentes necesitan que los padres alienten la autonomía y los apoyen. En cuanto a las relaciones interpersonales, los adolescentes necesitan vincularse con sus pares, sentirse aceptados e integrados al grupo; por lo que necesitan una inteligencia emocional adecuada para poder establecer estos vínculos y conformar su identidad. Al no lograr resolver de manera exitosa la elección de la ocupación, adopción de valores y el desarrollo de una identidad sexual satisfactoria; los adolescentes pasan a la crisis de identidad; la cual se caracteriza por una baja autoestima, conductas agresivas, sentimientos de exclusión y un auto concepto negativo (2013, p.67).

El análisis emocional de la autoconciencia es trascendental en la vida de las personas y los adolescentes no siempre logran dominar su conciencianisaberlasemocionesqueexperimentan. Si en la escuela esta competencia emocional es conocida y practicada convenientemente, o contemplada en algún contenido que tenga a bien el desarrollo humano, ésta puede ser de gran beneficio para el desarrollo integral y sostenido de los estudiantes. 


\section{MÉTODO}

Se asistió de una entrevista semiestructurada compuesta por 15 preguntas abiertas, referidas a la caracterización del desarrollo de la inteligencia emocional. Las primeras 4 preguntas orientadas a la primera dimensión que es el área intrapersonal, de la pregunta número 5 a la 8 orientadas a la segunda dimensión que es el área interpersonal, las preguntas del 9 al 12 orientadas a la tercera dimensión que es la adaptabilidad y por último las preguntas de la 13 a la 15 orientadas a la cuarta dimensión que es el estado de ánimo en general. La misma se aplicó a 30 estudiantes de ambos sexos, con edades comprendidas entre 16 y 17 años. Se contó con la experiencia de una trabajadora social y de una Psicóloga del establecimiento, quienes teniendo contacto directo con los adolescentes coadyuvaron con el desarrollo del proceso.

La guía de observación se realizó por el lapso de 10 días en la Unidad Educativa Tomás Frías, más propiamente en el 5to blanco, siendo la observación dentro y fuera del aula.

A continuación se presenta Tabla 1 colector del comportamiento de las variables, de las dimensiones y una serie de ítems indicadores del objeto de estudio en la investigación.

Tabla 1. Comportamiento de las variables.

\begin{tabular}{|c|c|c|c|c|c|c|}
\hline $\begin{array}{l}\text { Variable } \\
\text { dependiente }\end{array}$ & $\begin{array}{l}\text { Definición } \\
\text { conceptual }\end{array}$ & Dimensiones & Indicadores & 䓵 & 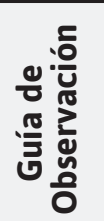 & $\stackrel{\check{g}}{\check{r}}$ \\
\hline \multirow[t]{9}{*}{$\begin{array}{l}\text { Inteligencia } \\
\text { emocional }\end{array}$} & $\begin{array}{l}\text { La inteligencia } \\
\text { emocional es la }\end{array}$ & $\begin{array}{c}\text { Área } \\
\text { Intrapersonal }\end{array}$ & ¿Reconoce sus emociones? & $x$ & $x$ & $x$ \\
\hline & $\begin{array}{l}\text { capacidad para } \\
\text { procesar la } \\
\text { información }\end{array}$ & & $\begin{array}{l}\text { ¿Comunica sus sentimientos a los } \\
\text { demás? }\end{array}$ & $x$ & $x$ & $x$ \\
\hline & $\begin{array}{l}\text { emocional con } \\
\text { exactitud y eficacia, } \\
\text { lo que incluiría }\end{array}$ & & ¿Reconoce sus logros? & $x$ & $\mathrm{x}$ & $x$ \\
\hline & $\begin{array}{l}\text { habilidades para } \\
\text { percibir, asimilar, } \\
\text { comprender y }\end{array}$ & & ¿Admite sus propios errores? & $\mathrm{x}$ & $x$ & $x$ \\
\hline & $\begin{array}{l}\text { regular, tanto las } \\
\text { emociones como los } \\
\text { estados de ánimo de } \\
\text { mayor duración. Este }\end{array}$ & & $\begin{array}{l}\text { ¿Toma sus propias decisiones } \\
\text { sin dejarse influenciar? }\end{array}$ & $x$ & $x$ & $x$ \\
\hline & $\begin{array}{l}\text { control o regulación } \\
\text { de las propias } \\
\text { emociones y estados } \\
\text { de ánimo ha sido }\end{array}$ & $\begin{array}{c}\text { Área } \\
\text { Interpersonal }\end{array}$ & $\begin{array}{l}\text { ¿Forma parte de un grupo de } \\
\text { amigos? }\end{array}$ & $x$ & $x$ & $x$ \\
\hline & $\begin{array}{l}\text { considerado por un } \\
\text { tercer component, } \\
\text { la empatía, que }\end{array}$ & & ¿Conversa con sus Compañeros? & $x$ & $x$ & $x$ \\
\hline & $\begin{array}{l}\text { completaría los dos } \\
\text { aspectos, afectivo y } \\
\text { cognitivo. }\end{array}$ & & $\begin{array}{l}\text { ¿Identifica los cambios de humor } \\
\text { que expresa la otra persona? }\end{array}$ & $\mathrm{x}$ & $x$ & $x$ \\
\hline & & & $\begin{array}{l}\text { ¿Respeta el estado anímico de su } \\
\text { compañeros? }\end{array}$ & $\mathrm{x}$ & $\mathrm{X}$ & $\mathrm{X}$ \\
\hline
\end{tabular}




\begin{tabular}{|c|c|c|c|c|c|c|}
\hline $\begin{array}{l}\text { Variable } \\
\text { dependiente }\end{array}$ & $\begin{array}{l}\text { Definición } \\
\text { conceptual }\end{array}$ & Dimensiones & Indicadores & & 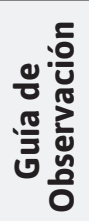 & $\stackrel{\breve{g}}{\leftarrow}$ \\
\hline \multirow[t]{6}{*}{$\begin{array}{l}\text { Inteligencia } \\
\text { emocional }\end{array}$} & $\begin{array}{l}\text { La inteligencia emocional } \\
\text { es la capacidad } \\
\text { para procesar la }\end{array}$ & \multirow[t]{3}{*}{ Adaptabilidad } & $\begin{array}{l}\text { ¿Plantea soluciones ante un } \\
\text { problema? }\end{array}$ & $x$ & $x$ & $x$ \\
\hline & $\begin{array}{l}\text { información emocional } \\
\text { con exactitud y } \\
\text { eficacia, lo que incluiría }\end{array}$ & & $\begin{array}{l}\text { ¿Se adapta a las reglas } \\
\text { del grupo? }\end{array}$ & $x$ & $x$ & $x$ \\
\hline & $\begin{array}{l}\text { asimilar, comprender } \\
\text { y regular, tanto las } \\
\text { emociones como los }\end{array}$ & & $\begin{array}{l}\text { ¿Colabora con las tareas } \\
\text { de los demás? }\end{array}$ & $x$ & $x$ & $x$ \\
\hline & $\begin{array}{l}\text { estados de ánimo de } \\
\text { mayor duración. Este } \\
\text { control o regulación de } \\
\text { las propias emociones y } \\
\text { estados de ánimo ha sido }\end{array}$ & \multirow[t]{3}{*}{$\begin{array}{l}\text { Estado de } \\
\text { ánimo general }\end{array}$} & $\begin{array}{l}\text { ¿Se acepta a si mismo tal } \\
\text { y cómo es? }\end{array}$ & $x$ & $x$ & $x$ \\
\hline & $\begin{array}{l}\text { considerado por un tercer } \\
\text { component, la empatía, } \\
\text { que completaría los } \\
\text { dos aspectos, afectivo y }\end{array}$ & & $\begin{array}{l}\text { ¿Se muestra positivo ante la } \\
\text { adversidad? }\end{array}$ & $x$ & $x$ & $x$ \\
\hline & & & $\begin{array}{l}\text { ¿Media en los conflictos con } \\
\text { los demás? }\end{array}$ & $x$ & $x$ & $x$ \\
\hline
\end{tabular}

\section{RESULTADOS}

\section{Dimensión: área intrapersonal}

Dentro de esta dimensión se evidencian indicadores que presentan mayores dificultades, en primera instancia está la falta de reconocimiento de los errores por parte de los adolescentes, donde se pudo observar que un $80 \%$ no lo hace. Seguido de las dificultades que tienen para demostrar sus emociones con un $67 \%$ Estos resultados indican que los adolescentes no asumen una posición autocrítica, que tienen dificultades para reflexionar sobre su propio comportamiento, como por ejemplo cuando el maestro llama la atención o sanciona al estudiante, éste no muestra ninguna reacción. (Gráfico 1).

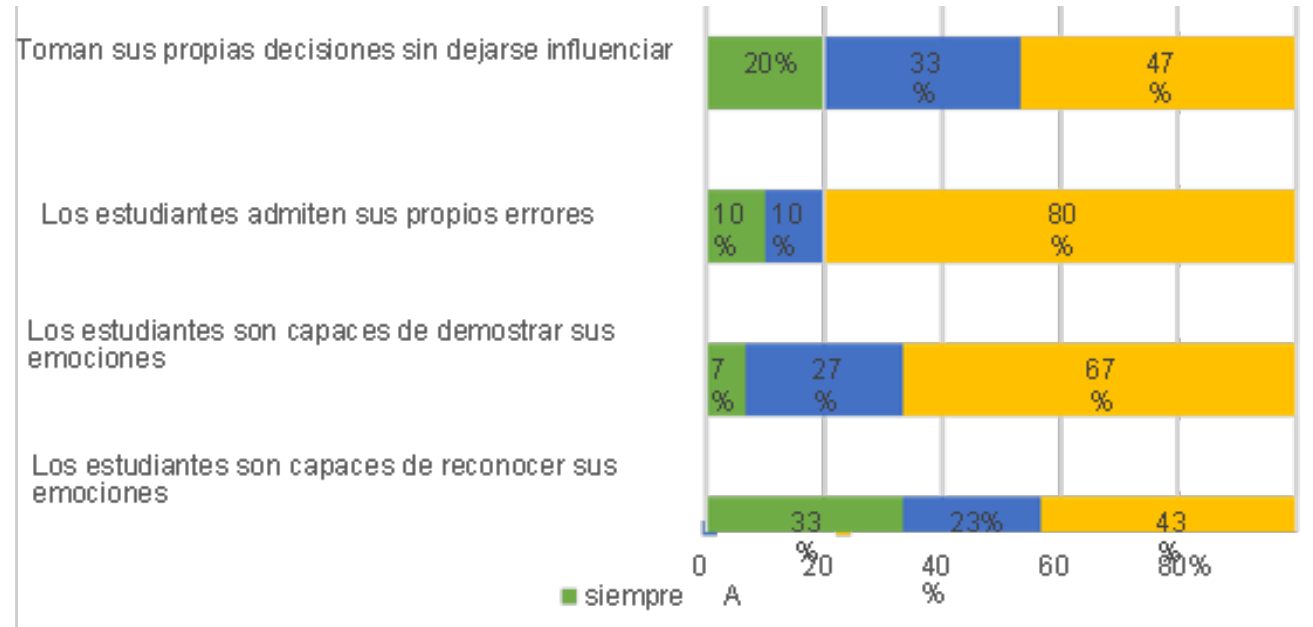

Gráfico 1. Área intrapersonal. 
Por otro lado, en situaciones donde se encuentra una figura que represente autoridad para los adolescentes, lo cual podría ser un factor que pueda cohibir el demostrar sus sentimientos, ellos muestran una actitud igualmente desinteresada en apariencia. Cuando un compañero molesta a otro, no expresa sus sentimientos, no dice estar enfadado, rabioso o feliz, solo se limitan a verbalizar palabras para que la otra persona deje su actitud del momento.

También se pudo observar que son pocos los adolescentes capaces de demostrar sus emociones, sobre todo si se trata de emociones negativas como enojo, rabia, ira y muchos menos el llanto. Los pocos momentos que alguno de los adolescentes muestra sus emociones lo hace de manera negativa, como por ejemplo pateando o tirando cosas, esto cuando no entran en acuerdo con los compañeros.

En el último ítem de esta dimensión se observó que los adolescentes no son capaces de tomar sus propias decisiones, lo hacen bajo la influencia de los demás compañeros; por ejemplo en el caso de las mujeres con la ropa y el maquillaje; preguntando a sus amigos si está bien, si le queda bien, si le habla o no le habla, si sale o no sale. El caso de los varones no está alejado de la misma realidad, para poder hablar a una chica, salir, hacer deporte o el mismo hecho de vestir la ropa lo hacen consultando a su entorno más cercano.

No se pudo observar en los adolescentes del 5 to blanco lo expuesto hasta el momento y analizado bajo lo que Gardner menciona del desarrollo de las inteligencias múltiples, haciendo referencia a la capacidad del acceso a la propia vida sentimental y de utilizarlos como un modo de comprender y guiar la conducta propia.

Una vez examinado todos los indicadores se analizó el indicador más significativo, no pueden reconocer sus propios errores. Siendo un $80 \%$ de la población estudiantil del 5to blanco, resulta realmente importante trabajar esta área, ya que este hecho a su vez se puede corroborar con lo manifestado por las profesionales del gabinete psicosocial. El hecho de culpar a los demás y no asumir la responsabilidad puede ser un factor que vienen arrastrando desde pequeños.

En relación a la primera dimensión denominada intrapersonal, ambas profesionales, psicóloga y trabajadora social, coincidieron al aseverar que la inteligencia emocional es el control que uno tiene al actuar, es decir, que uno primero debe pensar antes de actuar, teniendo un control sobre sí mismo. Ellas manifiestan que al momento de expresar sus emociones los estudiantes lo hacen de diferentes maneras, algunos patean algún objeto que tienen en frente, otros lloran; existiendo diversas maneras gestuales y corporales de expresar sus emociones y no así dando a conocer la emoción que están sintiendo al momento.

Respecto a la toma de decisiones la psicóloga quien es la que más trabaja el tema manifiesta que particularmente en esta gestión, se dio una ruptura en cuanto a la toma de decisiones como curso. Se vio segmentado influyendo de manera significativa ya que para las actividades de curso si bien los adolescentes daban su opinión al respecto, un grupo toma la decisión sin considerar la opinión del otro. A pesar de haberse trabajado esta situación sigue existiendo esa dificultad dentro este curso.

Por otro lado, también manifestaron que con esta gestión se tuvo bastantes problemas con los adolescentes, ya que hablando en la parte académica cuando suele pasar algún inconveniente los adolescentes culpan a los maestros, refiriendo que el docente la agarró con el grupo. Lo mismo pasa cuando existe alguna eventualidad entre estudiantes, se culpan entre sí llegando a negar no solo los aspectos negativos de su personalidad si no también los positivos. 


\section{Dimensión: área interpersonal}

El ítem más significativo dentro de esta dimensión es el respeto hacia las emociones de sus compañeros, representa el $80 \%$ de la población. Resultan valiosos estos datos ya que si bien reconocer las emociones propias es algo importante, esto va acompañado de la expresión y el respeto que tengan los demás compañeros a lo que expresan. De nada sirve reconocer sus propias emociones y expresarlas acordemente si estas no son respetadas por los otros. Lo ideal sería esforzarse en paralelo para lograr el objetivo comunicacional deseado. (Gráfico 2).

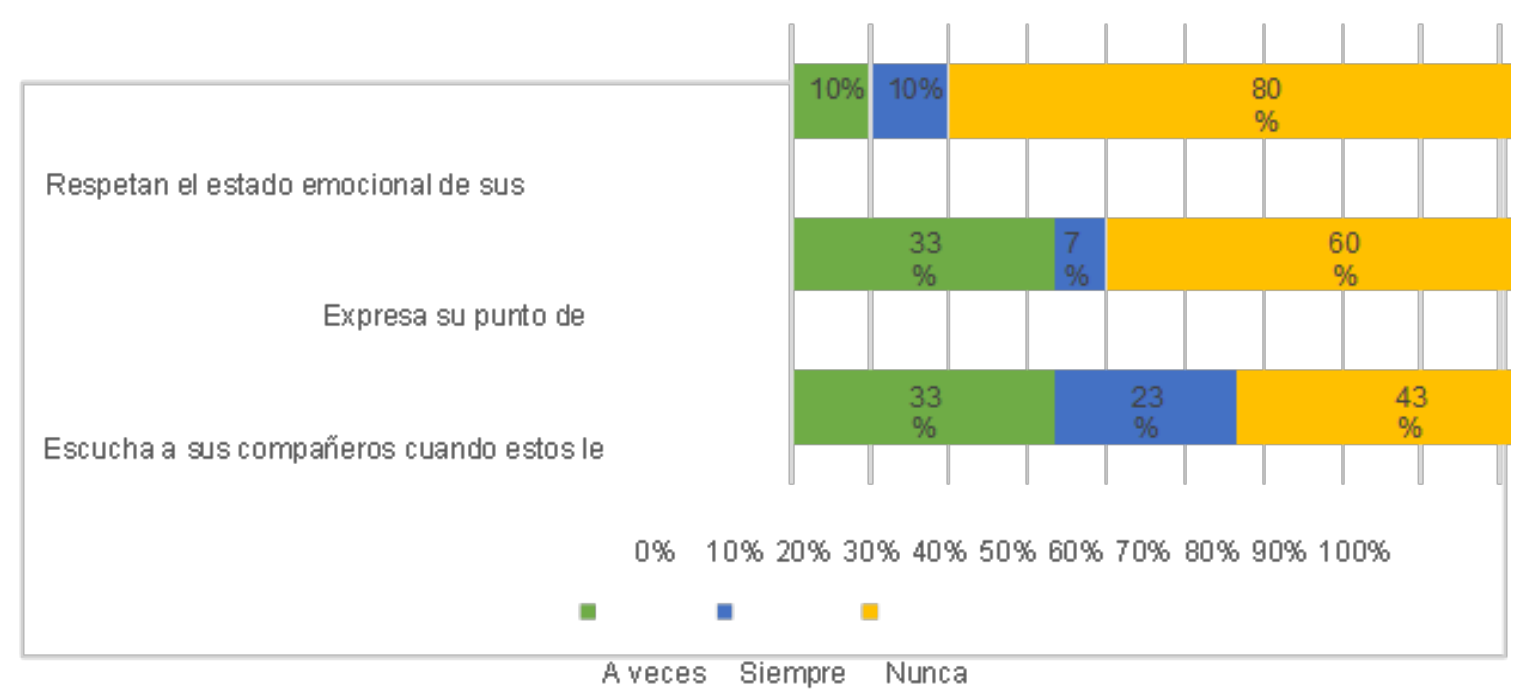

Gráfico 2. Área intrapersonal.

En el siguiente ítem que observa si el estudiante expresa su punto de vista, se evidenció que el $60 \%$ de los adolescentes expresan o quieren expresar su punto de vista, actitud que no siempre es respetada por el grupo. Haciendo relación con el ítem anterior existe una interferencia al momento que el adolescente está dando su punto de vista, están los celulares de por medio, compañeros con audífonos o hablando con terceras personas.

Lo anteriormente mencionado, pudo ser una razón muy fuerte por la que el resto de los adolescentes no quisieron verter opinión sobre un tema en particular, o expresar su punto de vista. Estando dentro del aula con algún maestro o autoridad estudiantil varía un poco la situación, pero no de manera significativa, ya que los celulares vuelven a ser la barrera, se dan modos de poder manejarlos sin que el profesor se dé cuenta, poniendo poca o nada de atención al estudiante que está hablando.

El último ítem si bien no llega al 50\% no deja de ser significativo. Ya que se pudo identificar que el escuchar a los compañeros cuando le hablan, es una conducta no practicada ya que casi todo el tiempo tienen puesto los audífonos o caso contrario están con el celular en la mano, existiendo una barrera comunicacional que impide que ellos desarrollen una comunicación efectiva. Los datos permiten apreciar que la mayor interferencia para la comunicación es la tecnología. 
En menor grado se observó que cuando un compañero le habla a otro siempre existe un tercero que interrumpe, de esta manera no se logra llegar a una buena conversación ya que el mensaje se ve interferido por elementos distractores.

En el aula se observa que existen grupos muy marcados lo que evidenció que existe dificultad entre ellos. Asociando la respuesta anterior cuando se menciona que hubo una ruptura a nivel de curso, esto dejo grupos con conflictos entre los mismos. Si un estudiante con pertenencia a un determinado grupo tiene algún problema con algún estudiante de otro grupo difícilmente se logrará la integración.

\section{Dimensión: adaptabilidad}

La adaptabilidad requiere dar soluciones ante un problema, situación que no se cumple en la mayoría de los adolescentes ya que cuando ocurre suelen asistir al gabinete psicosocial para encontrar alguna solución. También se ven consultando entre ellos las acciones que podrían tomar al respecto para posibilitar un desenvolvimiento correcto de las cosas. (Gráfico 3).

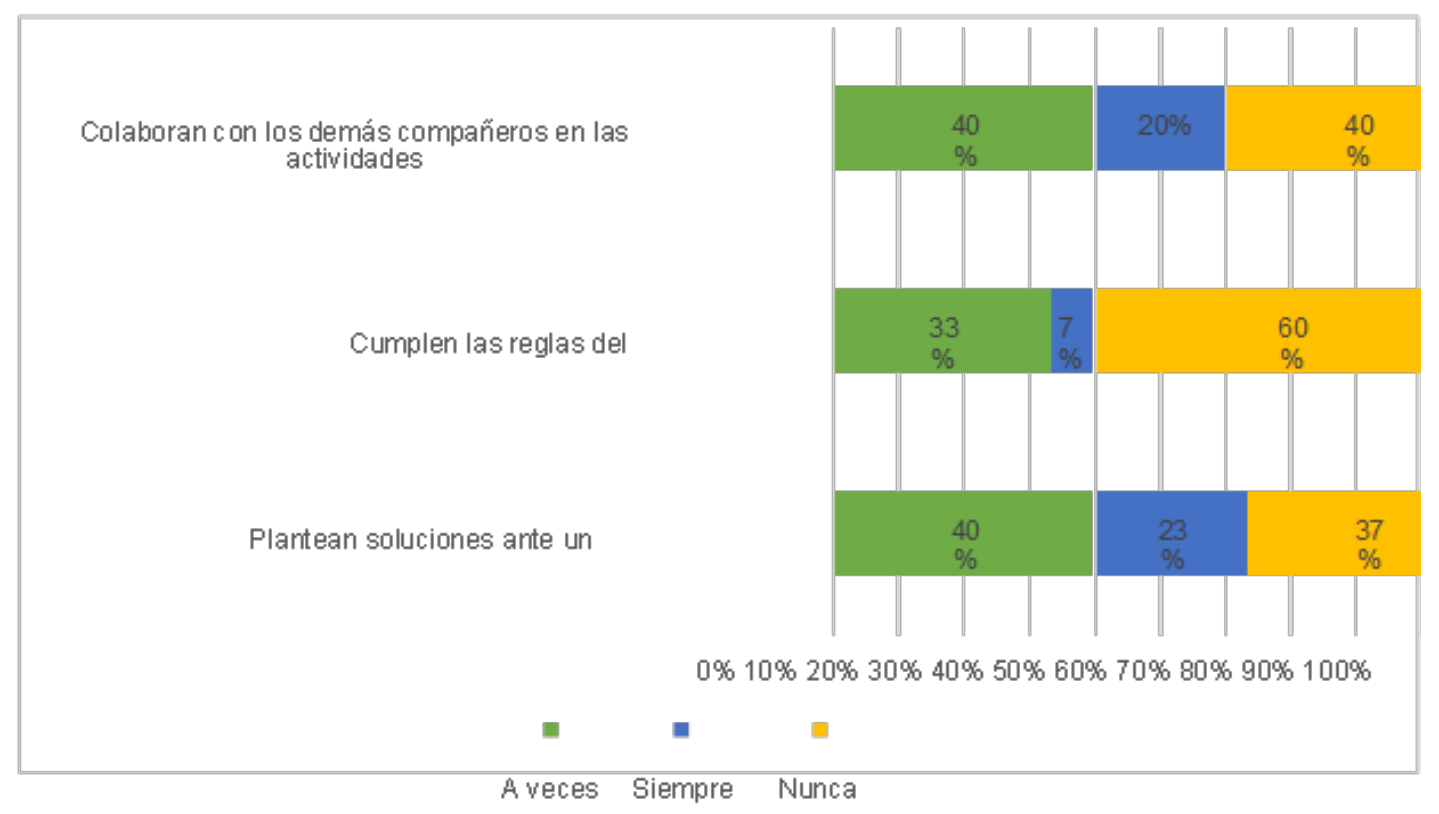

Gráfico 3. Área adaptabilidad.

En el ítem más significativo con el $60 \%$ cumplir las reglas del grupo, se observó que en su totalidad los adolescentes no llegan a hacerlo. Por ejemplo, el hecho de no estar callado mientras un compañero está hablando denota la falta de respeto que existe entre ellos.

El hecho de que no puedan respetar esta acción evita que los adolescentes puedan dar su opinión respecto alguna situación. Se pudo evidenciar poco el ítem la colaboración entre compañeros, ya que dentro del aula existen grupos muy marcados y si la actividad dirigida a trabajar no fuese del grupo de pertenencia, se muestra bastante apatía e inclusive intolerancia entre los mismos. A esto se suma que dentro del mismo grupo de pertenencia existe desorganización, desencadenando una falta de ayuda y de cooperación entre pares. 
Solo en determinados momentos reconocen cuando alguien está triste y tratan de cooperar hurgando las causas para hacerle sentir mejor.

En lo que respecta a contribuir con una solución ante un problema, generalmente recurren por ayuda al gabinete psicosocial o a su asesora.

\section{Dimensión: estado de ánimo general}

Con relación a esta dimensión la teoría manifiesta que muchas veces los adolescentes se dejan llevar por sus impulsos, ya que esto no está alejado de cómo piensan o de cómo hacen las cosas. Quizás se deba a los cambios que experimentan durante esta etapa tan compleja, como lo fisiológico, lo psicológico o lo social. Es en esta etapa donde el adolescente experimenta o vive emociones más intensas, es por ello que debe estar preparado emocionalmente para saber afrontar cualquier situación que se le presente y saber tomar las decisiones convenientes y adecuadas. (Gráfico 4).

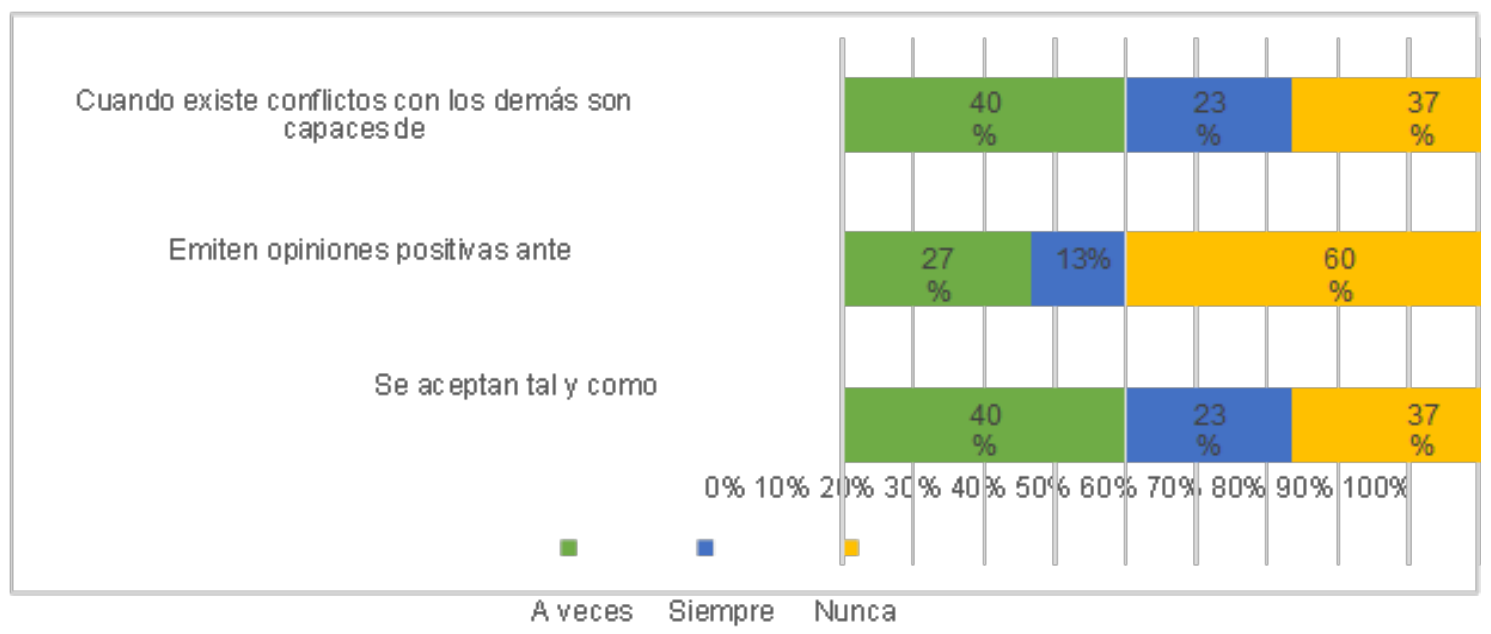

Gráfico 4. Estado de ánimo general

Las observaciones evidenciaron que los adolescentes no emiten opiniones positivas, al contrario las verbalizaciones que realizan entre ellos son negativas, emitiendo palabras ofensivas como sonso, burro, tonto, etc. Puede ser que motivado a estas palabras constantes emitidas por los compañeros que el estudiante no emite ningún tipo de emoción.

Haciendo la consulta a las profesionales del gabinete psicosocial, mencionan que solo en ocasiones se muestran optimistas ante las adversidades. Por lo general siempre están negativos ante un determinado problema debido a la segmentación de los grupos. A causa de esto se trató de integrarlos realizando talleres y charlas de manera grupal e individual, pero hasta el momento lo que se trabajo fue efímero consiguiendo que esta intervención resulte solo por un lapso de tiempo.

Ante la consulta si los estudiantes puedan expresar sus sentimientos de manera positiva la respuesta fue no. Argumentaron que algunos estudiantes vienen con problemas suscitados en la casa y no verbalizan esta situación, al contrario, lo que hacen es cambiar su conducta para que de esta manera llamen a los familiares para que intervengan, buscando también de cierta forma la atención de los padres. 
La situación entre pares no varía mucho al momento de manifestar sus emociones, prefieren hacerlo con conductas que hagan que la otra persona, lejos de reconocer su emoción, genere otra carga emocional.

Establecer metas y poseer autodisciplina permite actuar de acuerdo a los intereses propios sin olvidar el trabajo cooperativo en forma efectiva, reconociendo y estableciendo distinciones entre personas, promocionando la comunicación verbal y no verbal y desarrollando empatía con otras personas.

A manera de resumen se puede señalar que en lo que concierne a la entrevista al gabinete psicosocial, los adolescentes del 5to blanco de secundaria no tienen desarrollada la inteligencia emocional. Es algo complejo que debe tratarse con seriedad para comprender mejor al ser humano y sus comportamientos con los demás, esto incluye la competencia social, es decir, las relaciones interpersonales.

La motivación en las personas es trascendental para seguir adelante, esa fuerza interna que empuja a ser mejores, se debe estimular en la juventud y enlazarla con la auto- regulación. Practicar el auto control no es tarea fácil, pero sin duda debe ser un ejercicio constante en cada una de las personas. En la medida en que el ser humano avanza en su crecimiento personal y espiritual, manifiesta su comportamiento para con los demás y consigo mismo.

La adolescencia es una etapa difícil para cada ser humano y es en ella donde se deben adquirir conocimientos, desarrollar inteligencia racional y emocional para construir una personalidad que lo marcará y distinguirá por el resto de su vida. Si no hay autoconocimiento y un autocontrol se pueden desencadenar situaciones complejas que deriven en acoso sexual, violencia física o verbal, robo o comportamientos desviados de la rectitud moral y del código de valores que la sociedad mantiene.

Las emociones son sin duda algo muy complejo con lo que se vive; el conocer más sobre ellas, da una gama de información valiosa que se debe usar para dirigir nuestra vida hacia un éxito individual y colectivo.

\section{CONCLUSIONES}

Hubo una comprensión de la inteligencia emocional de acuerdo con los fundamentos teóricos y partiendo del objeto de estudio que son las inteligencias múltiples (intrapersonal e interpersonal) de donde se sustentó teórica y metodológicamente la inteligencia emocional. Entendida como la capacidad para procesar la información con exactitud y eficacia, lo que incluiría habilidades para percibir, asimilar, comprender y regular, tanto las emociones como los estados de ánimo de mayor duración en sí mismo y en el otro.

El diagnóstico realizado mediante los instrumentos empíricos aplicados dio a conocer la caracterización actual de la inteligencia emocional de los estudiantes, siendo las dimensiones que mayor dificultad presentan, la interpersonal y la intrapersonal. Identificando dificultades en los adolescentes para expresar sus emociones, reconocer las emociones de los demás, comunicar sus sentimientos, etc. Factores relevantes que deben ser trabajados para un adecuado desarrollo de la inteligencia emocional.

Tomando en cuenta la existencia de dificultades en cuanto al inadecuado manejo de la inteligencia emocional, se propone un programa educativo para desarrollar la inteligencia emocional en adolescentes del 5to blanco de secundaria, de la Unidad Educativa Tomás Frías de la ciudad de Potosí. 


\section{REFERENCIAS}

Álvarez, M., Bisquerra, R., Fita, E., Martínez, F., y Pérez, N., Evaluación de Programas de Educación Emocional. Revista de Investigación Educativa, 18 (2), 587-599

Bisquerra. (2013). Educación emocional. Barcelona: Barcelona, 67

Díaz Barriga, F., y Hernández Rojas, G. (1999). Estrategia docentes para un aprendizaje significativo. D.F: McGraw Hill.85

Espinar, R. (1993). Estrategias para trabajar. España: Mursia, 75

Fernández, A. (2011). Manual de inteligencia emocional. Colombia: Barbaos, 89

Gardner, H. (2001). Teoría de las inteligencias múltiples. Bogotá: FCE. 5

Goleman, D. (1998). Inteligencia emocional. Barcelona.125

Goleman, D. (1998). La práctica de la inteligencia emocional. Barcelona: Kairós. 196

Goleman, D. (2000). Inteligencia Emocional: Por qué es más importante que el cociente intelectual. Buenos Aires: B. 13
Gutiérrez, P. (2006). Diccionario Pedagógico. La Paz Bolivia: Gráficas, 89

Jacson, D. (2006). Inteligencia emocional en niños y adolescentes. España: Madrid. 14

Mesa, J. (2001). Atención de la inteligencia emocional. Disclouse. 15

Pajares, U. (2005). Inteligencia contra inteligencia emocional. México: Maya., 78

Papalia, M. (2009) Desarrollo Humano. México D.F. México: Mc Graw Hill Educación

Pérez, N. (2000). Revista de investigación educativa. España: Barcelona, p. 268

Cruz Roja, F. I. (2012). Planificación de proyectos y programas. Ginebra

Romero, Santos, C. (2007). Estrategias pedagógicas.

Salovey, M. (2007). Inteligencia emocional en adolescentes. Madrid.

Santos, B. (2000). Elaboración de videos. Cuzco: Lampayen 45

Unicef.(2002)Adolescencia una etapa fundamental. Nueva York: División de comunicaciones 